\title{
Endodontic procedures produce bacterial droplet contamination - but what about viruses?
}

\author{
Sabrina R. Rasaiah, ${ }^{1}$ James Allison ${ }^{2}$ and David C. Edwards ${ }^{* 3}$
}

\section{A commentary on}

\section{Bahador M, Alfirdous R A, Alquria T A, Griffin I L, Tordik P A,} Martinho F C.

Aerosols generated during endodontic treatments: a special concern during COVID-19 pandemic. J Endod 2021; 47: 732-739.

\begin{abstract}
Research question The study aims to investigate spread, distance and spatial distribution of aerosolised microorganisms generated through various endodontic procedures.

Study design A case-control study carried out at the University of Maryland, US in 2020. The study investigated the aerosolised microorganisms produced during three endodontic treatments: emergency pulpotomy, emergency pulpectomy and non-surgical root canal therapy (NSRCT), with 15 participants in each group (n $=45$ ). Patients diagnosed with symptomatic apical periodontitis were included. The use of settle plates for passive air sampling was employed, in a $4 \times 4 \mathrm{~m}$ room with closed doors. The number of colony-forming units (CFUs) and composition of bacterial species were analysed. Variables within the study included: distance of plate to patient's mouth $(0.5 \mathrm{~m}$ or $2 \mathrm{~m})$, positioning of plate (directly in front of or diagonal to the participant's mouth), type of endodontic treatment performed and the duration of treatment. A baseline
\end{abstract} sample was collected (room at rest) as a control. All dental treatment was carried out under rubber dam, after patients had undergone a pre-operative $0.12 \%$ chlorhexidine digluconate mouth rinse for 60 seconds. An extraoral suction system (ADS EOS Dental System Inc; Ontario, CA, USA) was also placed directly in front of the patient's mouth throughout treatment.

Results A significantly greater number of CFUs were recorded after endodontic treatments (all treatments), compared to negative control. CFUs were lower in the emergency pulpotomy group compared to the other two groups. There was a significantly lower number of CFUs found at plates situated $0.5 \mathrm{~m}$, compared to $2 \mathrm{~m}$, away from the patient. There was a positive correlation between the procedure duration and CFU count. The main bacterial species detected were Staphylococcus aureus (48.8\%), Staphlococcus epidermidis (42.2\%) and oral streptococci (33.1\%). Pseudomonas aeruginosa and fungi were not detected.

Conclusion Emergency pulpectomy and NSRCT created greater microbial contamination than an emergency pulpotomy. Greater numbers of microorganisms were found after longer treatment times and closer to the patient's mouth.

\section{Practice points}

- Bacterial contamination during endodontic procedures is highest closest to the patient's mouth.

- Bacterial contamination increases as the length of a procedure increases.

- This case-control study does not add to our knowledge of viral bioaerosol generation during endodontic procedures.

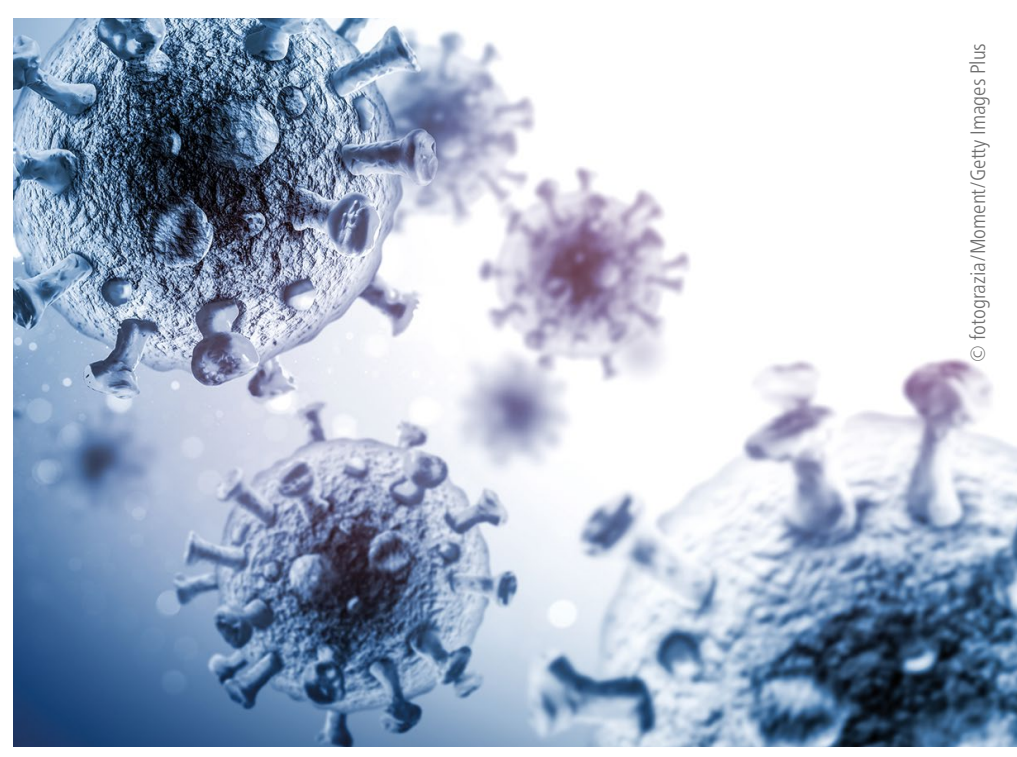

\section{Commentary}

SARS-COV-2 is present in significant quantities in saliva. ${ }^{1}$ Consequently, any dental procedure using a rotary handpiece inadvertently risks the spread of the virus through aerosols or droplets, posing a threat to both patients and clinicians. The lack of evidence on this topic ${ }^{2}$ resulted in guidance to stop performing aerosol generating procedures (AGPs) at the start of the COVID19 pandemic. Standard operating procedures are continuously evolving as new data and equipment are produced, which are facilitating dentistry's return to something that resembles 'normal practice'.

In the introduction of this paper, the authors demonstrate a compelling argument for the need of the study, particularly specific to the field of endodontics. Even when dentistry is restricted to urgent care, aerosol generating endodontic procedures remain essential to relieve patients' pain and infection.

The methods used in the study did not completely align with its stated aims. The authors used passive sampling with settle plates, which is reliable for detecting large droplets but has limitations for detecting aerosols; active sampling methods may have been more appropriate. ${ }^{3}$ Both 'procedure' and 'time' are stated as variables, but these are not investigated independently. Furthermore, the study aimed to identify the distance that bioaerosols can spread, but only investigated microorganisms at $0.5 \mathrm{~m}$ and $2 \mathrm{~m}$ and 
there is evidence that aerosols may travel beyond this. ${ }^{4}$ Spatial distribution is not robustly investigated as only two sampling points are used. Further limitations in the methodology include a lack of information on the flow rate of suction equipment and no information about ventilation rates within the surgery. A power calculation is not presented, making it unclear if the lack of significance of some results could represent a type II error.

Limitations to the external validity of this study for UK dentistry include the use of handpieces operating at 40,000 revolutions per minute (rpm). The use of high-speed instruments exceeding 60,000 rpm is classed as an AGP, with those under 60,000 rpm thought to result in minimal aerosol production. ${ }^{5}$ Conclusions from this study may have little impact on UK practice, as by definition, they only measure large droplet contamination and therefore should not be extrapolated to equipment that may create aerosols, producing droplets with particle sizes smaller than $5 \mu \mathrm{m}$ (for example, handpieces operating at 60,000 rpm). ${ }^{5}$

Another limitation to translation of findings to UK practice is the cross-infection control and aerosol mitigation measures used. The ADS EOS extraoral suction system is not widely used, and without knowing the flow rate, it is hard to interpret the likely impact of this measure. Room ventilation rates are not presented and this has significant implications on the mitigation measures required. ${ }^{5}$

The authors conclude that pulpotomy produces the least amount of 'aerosol'. As this was the shortest procedure, it is possible that duration, rather than procedure, is the most important variable. This is something which cannot be determined using the stated methodology. The paper suggests that the findings are concerning during the current COVID-19 pandemic; however, the methodology measures bacterial contamination in large droplets during endodontic procedures, not viral dispersion in bioaerosols. The findings are therefore of limited significance to the COVID-19 pandemic.

In summary, this is an interesting paper investigating bacterial spread, largely via large droplets, during endodontic procedures. However, the methodology limits the external validity and the results do not necessarily relate to the generation of aerosols containing viruses.

\section{Author affiliations}

${ }^{1}$ General Professional Trainee, Newcastle University School of Dental Sciences, Newcastle Upon Tyne, UK; ${ }^{2}$ Clinical Fellow in Oral Surgery, Newcastle University School of Dental Sciences, Newcastle Upon Tyne, UK; ${ }^{3}$ Academic Clinical Fellow and Honorary Speciality Trainee in Endodontics, Newcastle University School of Dental Sciences, Newcastle Upon Tyne, UK. *Correspondence to: David Edwards

\section{References}

1. Riccò M, Ranzieri S, Peruzzi S et al. RT-qPCR assays based on saliva rather than on nasopharyngeal swabs are possible but should be interpreted with caution: results from a systematic review and meta-analysis. Acta Biomed 2020; DOI: 10.23750/ abm.v91i3.10020.

2. Innes $\mathrm{N}$, Johnson I G, Al-Yaseen $\mathrm{W}$ et al. A systematic review of droplet and aerosol generation in dentistry. J Dent 2020; 23: 103556

3. Zemouri C, Volgenant C M, Buijs M J et al. Dental aerosols: microbial composition and spatial distribution. J Oral Microbiol 2020; DOI: 10.1080/20002297.2020.1762040.

4. Holliday R, Allison J R, Currie C C et al. Evaluating contaminated dental aerosol and splatter in an open plan clinic environment: Implications for the COVID-19 pandemic. J Dent 2021; 105: 103565.

5. Public Health England. COVID-19: infection prevention and control dental appendix. 2020. Available at https://assets.publishing.service.gov.uk/government/ uploads/system/uploads/attachment_data/file/965686/Infection_prevention_and_ control_guidance_Dental_appendix_.pdf (accessed March 2021).

Evidence-Based Dentistry (2021) 22, 76-77.

https://doi.org/ 10.1038/s41432-021-0175-1 